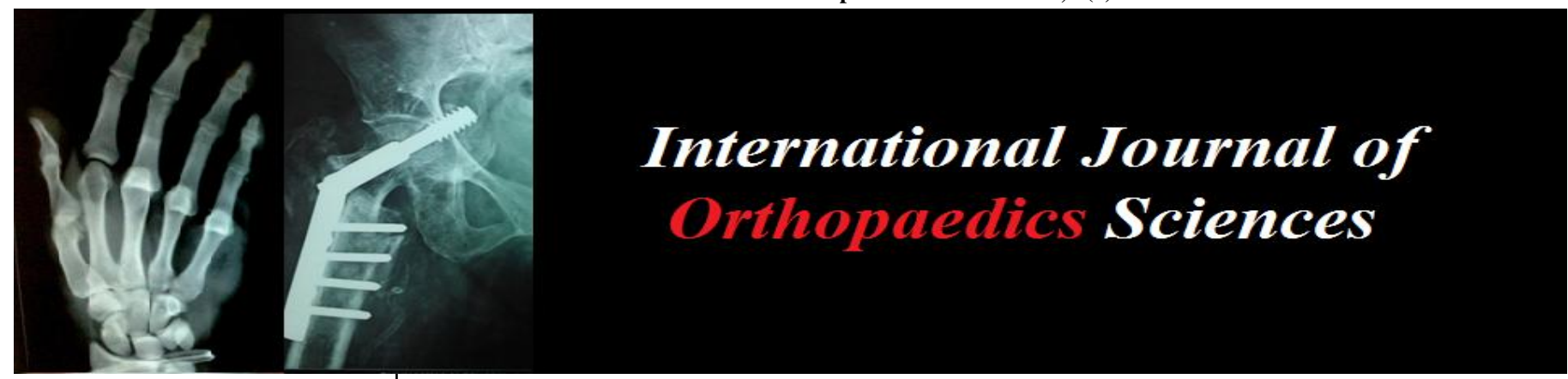

ISSN: $2395-1958$

IJOS 2018; 4(4): 644-648

(C) 2018 IJOS

www.orthopaper.com

Received: 15-08-2018

Accepted: 20-09-2018

\section{Senthuren Isaac}

MBBS, Royal Perth Hospital, 197 Wellington Street, Perth

WA, Australia

Zachary Sinagra

MBBS, BSc, Royal Perth

Hospital, 197 Wellington Street,

Perth WA, Australia

\section{Alfredo Pineda}

MD, Royal Perth Hospital, 197

Wellington Street, Perth WA,

Australia

\section{Nicholas Grainger}

Royal Perth Hospital, 197

Wellington Street, Perth WA,

Australia

\section{Mark Hurworth}

Royal Perth Hospital, 197

Wellington Street, Perth WA,

Australia
Correspondence

Zachary Sinagra

MBBS, BSc, Royal Perth

Hospital, 197 Wellington Street,

Perth WA, Australia

\section{Comparing porcine models and sawbones as a training tool for complex arthroscopic knee surgery}

\author{
Senthuren Isaac, Zachary Sinagra, Alfredo Pineda, Nicholas Grainger \\ and Mark Hurworth
}

DOI: https://doi.org/10.22271/ortho.2018.v4.i4h.77

\section{Abstract}

Background: Anterior cruciate ligament (ACL) reconstruction and meniscal repair are two commonly performed arthroscopic soft tissue surgeries. It can be challenging for training surgeons to become proficient in these techniques. The aim of our study is to investigate the viability of porcine stifles as training models for ACL reconstruction and meniscal repair.

Method: A training session was held with various participants comparing the use of porcine stifles vs. sawbone replicas. Tendon harvesting, joint arthroscopy, meniscal repair and ACL reconstruction were performed on both groups of joints. The two training methods were compared by the completion of a questionnaire by four participants.

Results: Porcine stifles were found to be a suitable alternative to human knee joints. Tactile sensation of performing these procedures with porcine stifles more closely resembled that of a human knee. Visualisation of the joint and its structures was considerably easier in the saw bone model, but the porcine stifle better resembled real life scenarios. The medial joint compartment was notably more difficult to visualise and access relative to the lateral compartment, increasing procedure time when repairing the medial meniscus.

Conclusion: Porcine stifles provide a suitable model for education and surgical training for ACL reconstruction and meniscal repair for trainee surgeons. They are easy to procure, cost-effective, and better replicate the complexities of these procedures in human knees compared to sawbones. We believe that practically this provides an ideal platform for simulation scenarios which are similar to humans and fulfils the ethical mandates of beneficence and non-maleficence.

Keywords: porcine models, sawbones, training tool, knee surgery

\section{Introduction}

Conventional teaching of trainee surgeons to perform arthroscopic procedures involves an apprenticeship model, including many hours of observation and assisting more senior surgeons. The complexity of these surgical procedures attaches a sharp learning curve, increasing the risks of surgical error and prolonged operative time. Knee injuries are common presentations to any Orthopaedic practice, with anterior cruciate ligament (ACL) ruptures and meniscal tears largely being seen among younger cohorts of patients, who typically have higher athletic and functional demands [1,2]. Surgical management of these injuries are technically challenging and should be undertaken by proficient surgeons.

Alternatives to the apprenticeship method include human cadaveric models and the use of virtual reality (VR) training systems. Cadaveric models provide the closest simulation to reallife procedures but are limited by factors such as significant cost and availability of models in adequate numbers ${ }^{[3]}$. VR systems provide a platform to practise the theory behind these procedures, however are limited by cost and the inability to provide realistic tactile simulation [3-5]. The use of anatomic dry models, such as sawbones, have also traditionally been used in arthroscopic training due to their cost effectiveness and reproducibility. They have been shown to be good models for procedural training, although are limited in that they do not provide the same realistic environment as cadaveric or animal platforms ${ }^{[6-8]}$.

With consideration of these available options and their limitations, animal models provide a potentially realistic and cost-effective alternative means for orthopaedic trainees to perform 
arthroscopic meniscal repair and ACL reconstruction in a controlled learning environment. A variety of different animal models have been previously utilised to perform and teach ACL reconstruction, such as described by Hunt et al., who utilised ovine models to perform open ACL reconstruction ${ }^{[9]}$. Similar studies have explored the use of various different animal models for biomechanical and tissue healing analysis of the anterior cruciate ligament. Kovac et al. have also previously explored the use of ovine, bovine and porcine models in the training of arthroscopic meniscal repair, with the latter being shown to be the most cost effective, accessible and comparatively similar alternative to the human knee ${ }^{[10]}$.

Here we present our experience in comparing porcine stifles to traditional sawbone artificial knee models in the teaching of arthroscopic ACL reconstruction and meniscal repair procedures.

\section{Materials and Methods}

The training session was conducted at the Large Animal Laboratory at Royal Perth Hospital, a centre that runs animalmodel based training programs. The laboratory provided porcine stifles, which are readily available due to the use of these models in other training programs. There were four participants involved in this study with their skill and experience levels ranging from junior registrar $(n=2)$, to orthopaedic fellow $(n=1)$ and senior consultant $(n=1)$.

Stifles underwent further preparation, involving removal of excess muscle and fat tissue, and release of tendons that cross the joint. The tendons crossing the adjacent joint were also released. This process allowed for deeper flexion of the stifle joint than would otherwise be possible (up to $140^{\circ}$ ) thus better recreating the intra-operative conditions and range of motion of a human knee joint. Stifles also had the hide removed, in order to improve the ease of access and reduce the likelihood of the excessive extra-articular material impeding the procedure.

The intramedullary canals of the porcine tibial bones were reamed using a standard household drill in order to allow for mounting on a custom-made metal jig. This jig comprised of a clamp attached to the training table with a metal rod orientated upwards for mounting of the porcine tibia via the reamed intramedullary canal. This method was also utilised in our previous studies involving the use of bovine carpal joints ${ }^{[11]}$. This provided a stationary platform upon which the model was seated during the training exercise (Figure 1). A $2.7 \mathrm{~mm}$ wrist arthroscope (ie. Smaller than a normal knee arthroscope) was used together with an analogue arthroscopy equipment stack, as this size best suited the dimensions of the porcine stifle. The sawbone models were set up in a standard fashion held in place by their commercially produced mounts (Figure 2).

In both models, a trochar and scope portal were introduced into the joint using the standard anterolateral portal positioning for a human knee $[1.5 \mathrm{~cm}$ above the lateral joint line in the lateral soft spot] with a second instrumentation portal located in the standard anteromedial position $[1.5 \mathrm{~cm}$ above the medial joint line in the medial soft spot] ${ }^{[12]}$. For the porcine stifles, a cautery device was used to debulk the intracapsular fat pad to improve the surgeon's vision. The arthroscopic landmarks of the porcine stifle closely resembled that of the human knee. A simple 'all-inside' meniscal repair and ACL reconstruction was performed by all participants on both sawbone and porcine models, using a commercially available system for each procedure (Figures 3 and 4). A standard household drill was used to create bone tunnels. The tendon grafts were harvested by sharp dissection from bovine wrist extensors (Figure 5.), which we have previously described as being the same size and calibre as human hamstring and provide a well-suited graft for the ACL reconstruction based on size and tactile feel (Figure 6.) ${ }^{[11]}$.

After the training modules were completed, participants answered questionnaires assessing their experience and thoughts on the two different teaching models. The questionnaire included nine questions inquiring about tactile sensation, accessibility and visualisation of the porcine joint, the theory and steps of both meniscal repair and ACL reconstruction, graft preparation, improvement in proficiency in performing each technique, effectiveness of the model and recommendations for use. Each question was assessed on a scale of "1 - strongly disagree" to "5 - strongly agree", with agreeing having been in favour of the porcine model compared to the sawbone model.

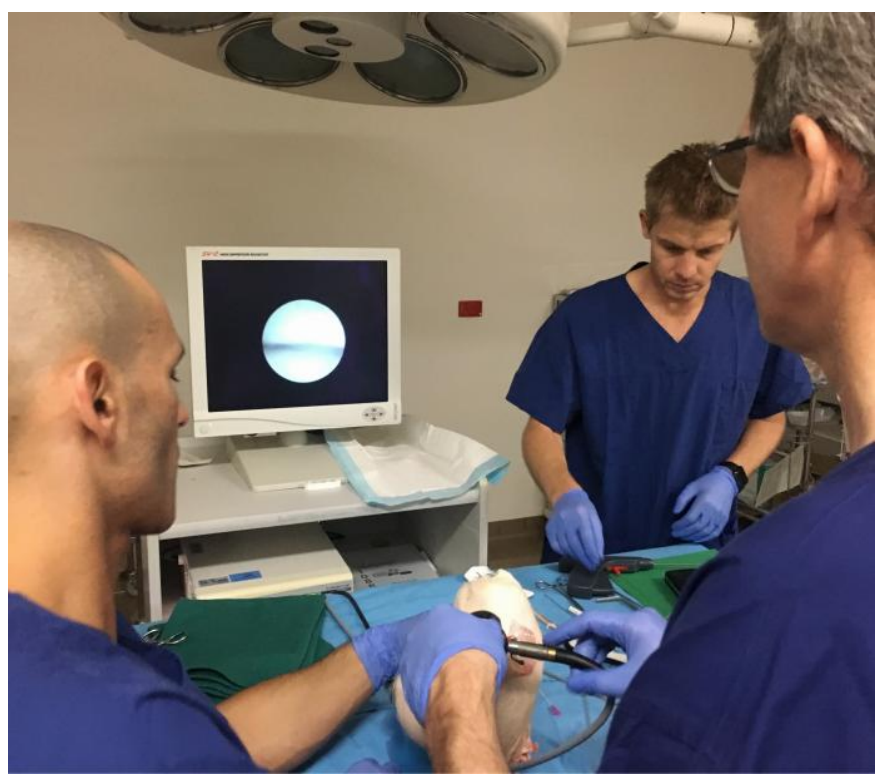

Fig 1: Porcine stifle preparation and setup.

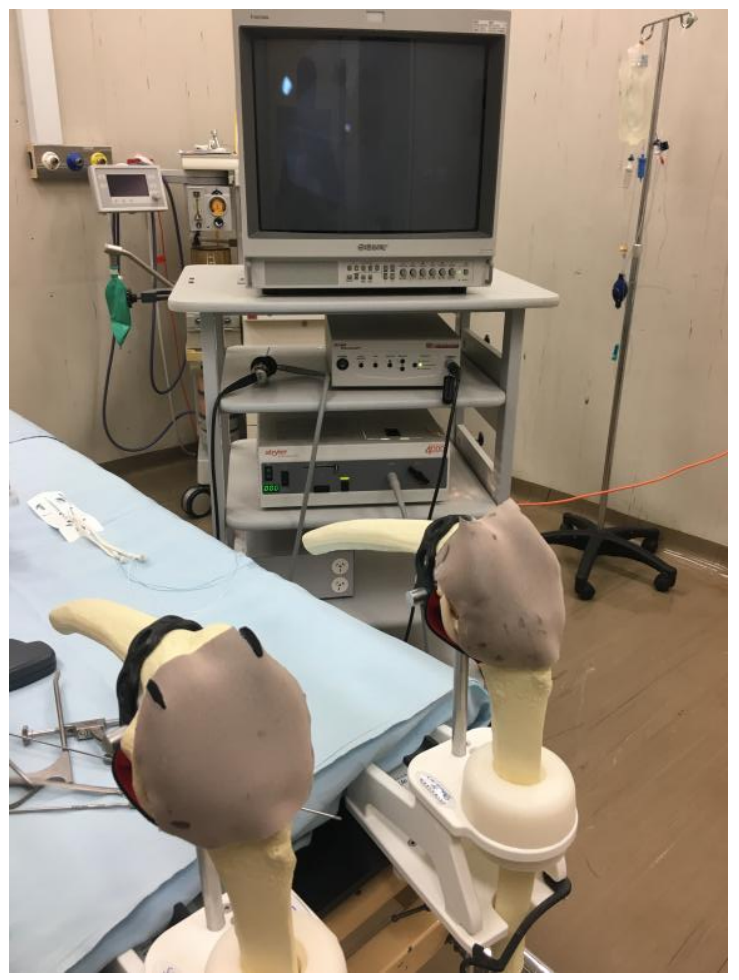

Fig 2: Sawbone model setup. 


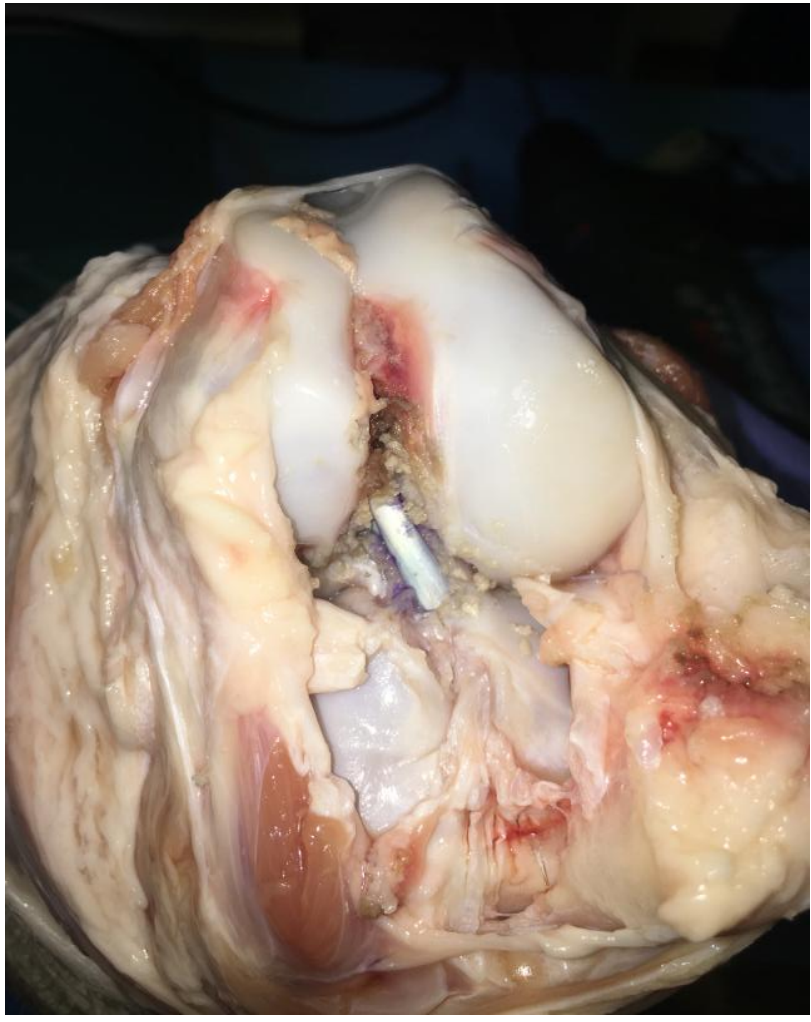

Fig 3: Porcine ACL reconstruction. After completion of the training, the porcine stifles were opened to access the ACL reconstruction.

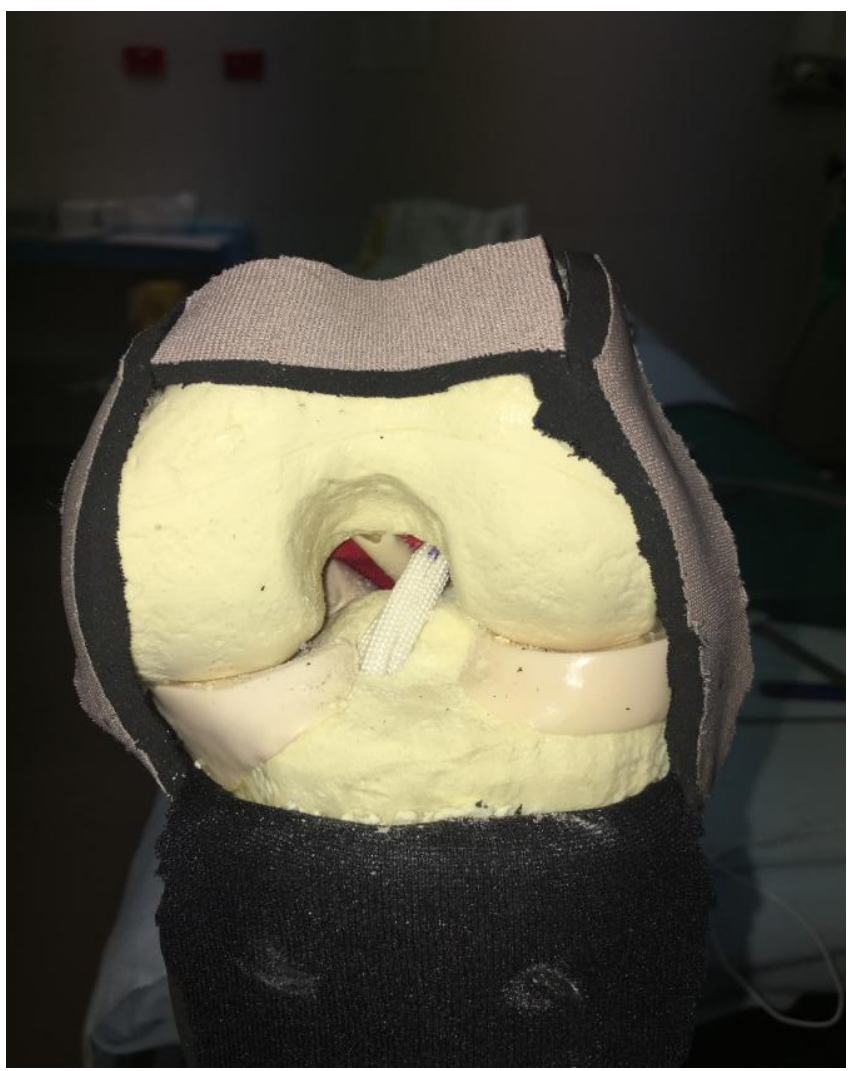

Fig 4: Sawbone ACL reconstruction and meniscal repair. The fabric cover has been removed upon completion of the procedure to inspect the results.

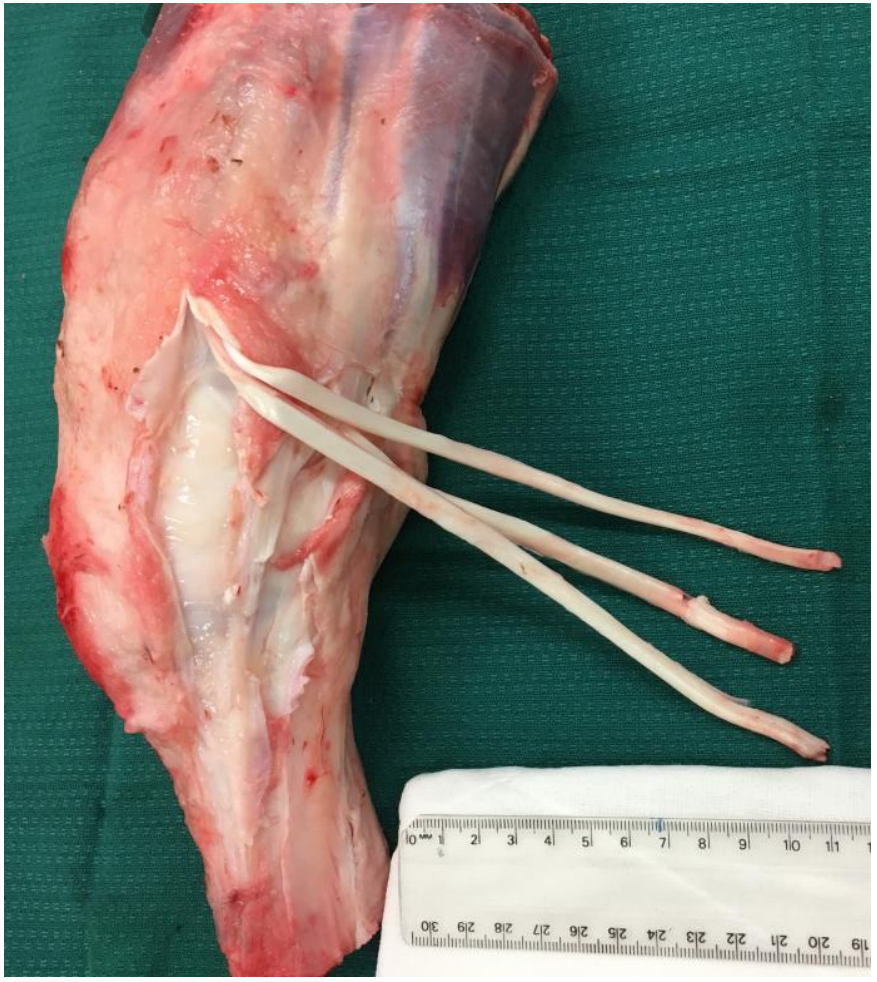

Fig 5: Harvesting of bovine extensor tendons.

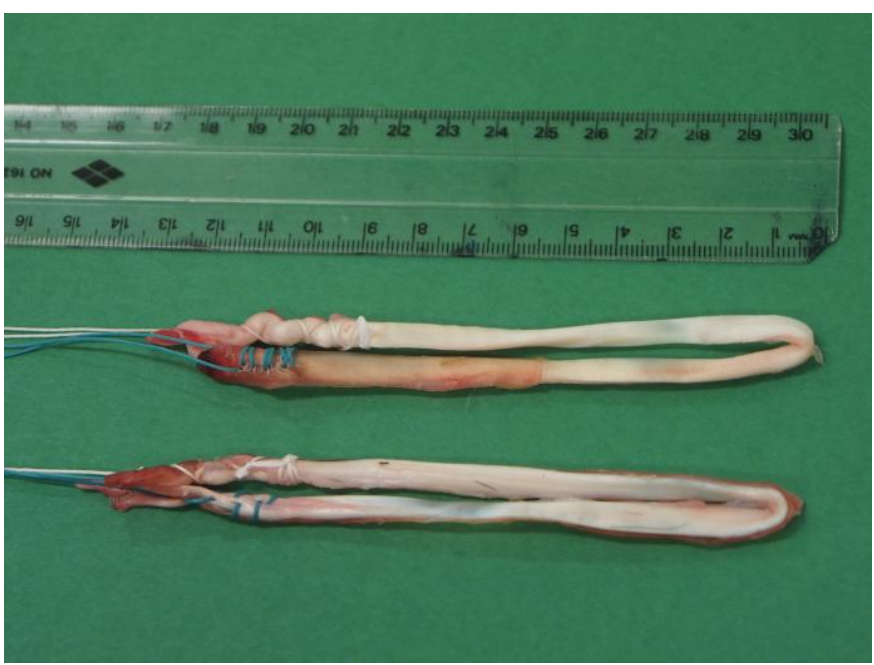

Fig 6: Prepared bovine tendon grafts prior to mounting on an endobutton loop.

\section{Results}

The first part of the questionnaire was concerned with the tactile sensation when first performing the diagnostic arthroscopy (Figure 7). The replies rated an average of 4.5, meaning that the tactile feedback for the porcine stifle better resembled that of a human knee. The accessibility and visualisation of the porcine joint produced and average response of 4.5 and 3.75 respectfully, indicating that the accessing of and manoeuvring inside the joint closely resembled a human knee arthroscopy, while the visualisation of the joint structures was similar, but not entirely the same. 
Tactile Sensation

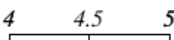

Accessibility of Joint

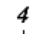

Visualisation of Joint

\begin{tabular}{llllll}
\hline \\
\hline
\end{tabular}

Fig 7: The impressions received from the responses regarding basic arthroscopy were in favour of the porcine model as a suitable alternative to the human knee when compared to the sawbone model.

Meniscal repair and ACL reconstruction procedures were undertaken on both porcine and sawbone models by each participant with the results outlined in Figure 8. Applying the theory and undertaking the physical steps of meniscal repair received a neutral response and was equal for both porcine and sawbone models, reflected by a mean score of 3 . ACL reconstruction received a mean score of 4 , indicating that performing this procedure on a porcine stifle was thought to be a better representation of the human operative scenario compared to the sawbone model. The preparation of the bovine extensor tendon graft involved in the porcine procedure was favoured compared to the graft preparation for the sawbone models as expressed by a mean feedback score of 4.5 out of 5 .
Meniscal Repair

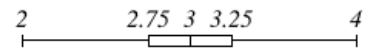

ACL Reconstruction

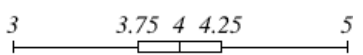

Graft Preparation

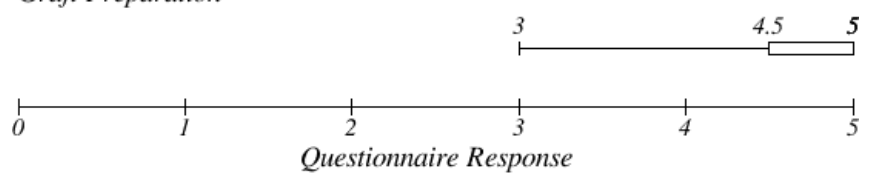

Fig 8: Feedback indicated that neither the porcine or sawbone models were superior training methods for the meniscal repair procedure. The graft preparation and ACL reconstruction proved to be more realistic and a better representation of the real-life human scenario in porcine stifles compared to the sawbone models.

The porcine model was felt to have the greater potential for gaining proficiency in performing each technique as the feedback questionnaire produced a mean of 4.75 in its favour versus that of the sawbone model (Figure 9). The overall effectiveness and cost efficiency was in favour of the porcine model, at a mean score of 4.25 . With a mean feedback score of 4.5, all participants involved in this study recommended the use of porcine stifles as a valid and effective arthroscopic training model for developing surgeons.

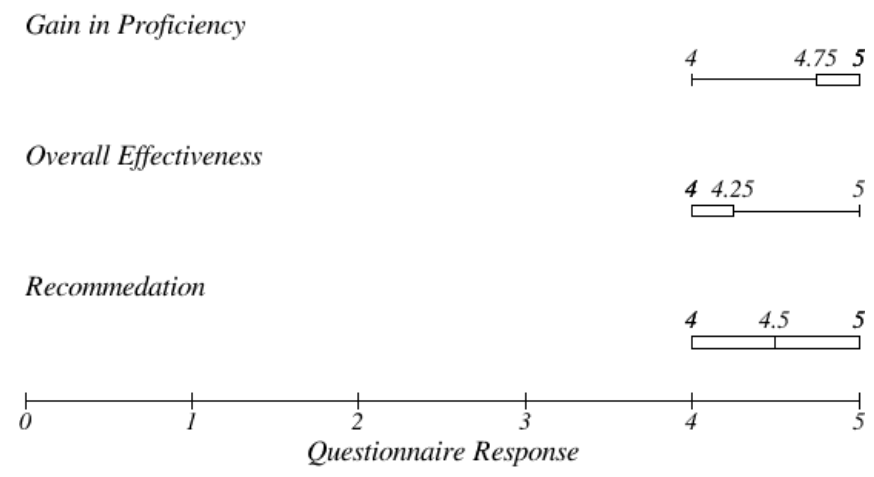

Fig 9: The porcine training model received better feedback regarding gain in proficiency and overall effectiveness than the sawbone model. All participants recommended the use of porcine stifles as a valid and effective arthroscopic training model for developing surgeons.

\section{Discussion}

Feedback received from the questionnaires indicated that porcine stifles were a suitable alternative to human knee joints. Once the thick musculature was debulked, the porcine stifle allowed for deeper flexion similar to human knee joints. Removal of the hide from the stifle enabled easy palpation of landmarks, as well as entry into the joint. The participants agreed that the porcine hide was significantly different to the human skin and did not provide any benefit to recreating the feel of a human knee. Tendons were easily harvested and of a similar in size and handling to those harvested from human hamstrings ${ }^{[11]}$ (Figure 6.).

The joint capsule was robust enough to allow for saline irrigation during arthroscopy, allowing for completion of both meniscal repair and ACL reconstruction. Tactile sensation of performing these procedures with porcine stifles more closely resembled that of a human knee, in comparison to saw bone models (Figure 7). Access to and visualisation of the joint was considerably easier in saw bone models, but the porcine stifle better resembled real life circumstances.
The porcine stifles did present the participants with some limitations. Amongst these were that they are a smaller size as the human knee joint, the medial joint compartment in the stifle was notably more difficult to visualise and access, relative to the lateral compartment. This resulted in participants taking longer to repair the meniscus. In addition, due to the large anterior fat pad we found that removal with a cautery device prior to commencing the meniscal repair and ACL reconstruction, significantly improved visibility and resulted in decreased time of procedure.

Presently surgical training across subspecialties follows an apprenticeship model, where trainees will assist the surgeon, and then gradually be allowed to perform aspects of the surgery themselves. Other disciplines which involve significant responsibility for human safety, such as aviation, make more use of simulation training, and such training remains mandatory on a regular basis, even for experienced personnel ${ }^{[13]}$. This training enables the trainee to practice on the difficult, unexpected or unusual scenarios, which places them in a satisfactory position should these conditions be 
encountered. We hold that this may be a useful model to be pursued for surgical training i.e. training in a more hostile than usual environment can only be good for all parties - both surgeons and patients. In theory, training in a more difficult environment will make the transition to a normal intraoperative environment easier, as well as prepare the operator for difficult situations where they encounter cases with poor visibility or suboptimal operating conditions.

With this in mind, it seems reasonable that surgical training encounters should be able to simulate the difficult, the unusual and the unexpected. Ideally, trainees and perhaps even experienced surgeons should train in "worst case" scenarios on a regular basis, similar to what is expected of pilots of passenger aircraft. It is difficult to envision a situation where sawbones, or even human cadavers, could be used for this purpose. Hence, an animal training model that simulates a difficult human knee would seem to be an ideal solution to what we see as an ethical and practical dilemma i.e. how dowe benefit our patients and surgeons, while doing least or preferably no harm (beneficence and nonmaleficence).

With this as background, we found porcine stifles to be a suitable alternative to human knee joints for arthroscopic training. The anatomy of the porcine stifle is similar to that of the human knee ${ }^{[14]}$, with a smaller and wider medial meniscus being the main difference. As such, porcine stifles provide a useful arthroscopic environment, similar to a human knee, for arthroscopic training. The experience of harvesting and preparing bovine graft tendons, as well as performing arthroscopy in porcine stifles, were both superior to sawbone training, providing a much closer representation to the human knee.

Although superior to sawbone models, the porcine stifles did present the authors with some limitations. Porcine stifles have relatively larger anterior fat pad, in comparison to human knees, which poses difficulties to vision and instrumentation. If not adequately debrided, this can restrict visibility throughout the procedure. The medial stifle joint compartment was notably more difficult to visualise and access, resulting in longer surgical times in repairing medial meniscal tears, with increased technical difficulty.

The ease of procurement (available from any butcher) and storage, along with the realistic tactile sensation achieved with porcine stifles and bovine wrist extensor tendons, makes them a readily available, safe and suitable method of training for surgeons in arthroscopic techniques.

\section{Conclusion}

Porcine stifles and bovine extensor tendons provide a suitable model for surgical education and training in anterior cruciate ligament reconstruction and meniscal repairs for trainee surgeons. They are easy to procure, cost-effective, and better replicate the complexities and intra-operative conditions of such procedures in human knees compared to saw bones. Additionally, as a training model, porcine stifles fulfil the ethical requirements of beneficence and non-maleficence.

\section{References}

1. Beynnon BD, Johnson RJ, Abate JA, Fleming BC, Nichols CE. Treatment of Anterior Cruciate Ligament Injuries, Part I. The American Journal of Sports Medicine. 2005; 33(10):1579-602.

2. Beynnon BD, Johnson RJ, Abate JA, Fleming BC, Nichols CE. Treatment of Anterior Cruciate Ligament Injuries, Part 2. The American Journal of Sports
Medicine. 2005; 33(11):1751-67.

3. Camp CL, Krych AJ, Stuart MJ, Regnier TD, Mills KM, Turner NS. Improving Resident Performance in Knee Arthroscopy: A Prospective Value Assessment of Simulators and Cadaveric Skills Laboratories. J Bone Joint Surg Am. 2016; 98(3):220-5.

4. Jacobsen ME, Andersen MJ, Hansen CO, Konge L. Testing basic competency in knee arthroscopy using a virtual reality simulator: exploring validity and reliability. J Bone Joint Surg Am. 2015; 97(9):775-81.

5. Peres L, Junior W, Coelho G, Lyra M. A new simulator model for knee arthroscopy procedures. Knee Surgery, Sports Traumatology, Arthroscopy. 2017; 25(10):307683.

6. Butler A, Olson T, Koehler R, Nicandri G. Do the skills acquired by novice surgeons using anatomic dry models transfer effectively to the task of diagnostic knee arthroscopy performed on cadaveric specimens? J Bone Joint Surg Am. 2013; 95(3):e15(1-8).

7. Hetaimish BM. Sawbones laboratory in orthopedic surgical training. Saudi Medical Journal. 2016; 37(4):348.

8. Dwyer T, Slade Shantz J, Chahal J, Wasserstein D, Schachar R, Kulasegaram KM, et al. Simulation of Anterior Cruciate Ligament Reconstruction in a Dry Model. The American Journal of Sports Medicine. 2015; 43(12):2997-3004.

9. Hunt P, Scheffler SU, Unterhauser FN, Weiler A. A model of soft-tissue graft anterior cruciate ligament reconstruction in sheep. Arch Orthop Trauma Surg. 2005; 125(4):238-48.

10. Kovac N, Grainger N, Hurworth M. Training models for meniscal repairs and small joint arthroscopy. ANZ J Surg. 2015; 85(9):649-51.

11. Calvert N, Grainger N, Hurworth M. Use of bovine carpal joints as a training model for cruciate ligament repair. ANZ J Surg. 2013; 83(12):933-6.

12. Hoppenfeld S, de Boer P, Buckley R. Surgical Exposures in Orthopaedics: The Anatomic Approach. 5th ed. Philadelphia, United States of America: Wolters Kluwer; 2016.

13. Taylor A, Dixon - Hardy D, Wright S. Simulation Training in U.K. General Aviation: An Undervalued Aid to Reducing Loss of Control Accidents. 2014; 141-52. ISSN 1050-8414.

14. Proffen BL, McElfresh M, Fleming BC, Murray MM. A comparative anatomical study of the human knee and six animal species. Knee. 2012; 19(4):493-9. 